\title{
Local Nonnegative Matrix Factorization for Mining Typical User Session Profile*
}

\author{
Jixiang Jiang ${ }^{1}$, Baowen $\mathrm{Xu}^{1,2^{* *}}$, Jianjiang $\mathrm{Lu}^{1,2,3}$, and Hongji Yang ${ }^{4}$ \\ ${ }^{1}$ Department of Computer Science and Engineering, Southeast University, \\ Nanjing, 210096, China \\ ${ }^{2}$ Jiangsu Institute of Software Quality, Nanjing, 210096, China \\ ${ }^{3}$ PLA University of Science and Technology, Nanjing 210007, China \\ ${ }^{4}$ School of Computing, De Montfort University, Leicester, LE1 9BH, England
}

\begin{abstract}
Understanding the evolving user session profile is key to maintaining service performance levels. Clustering techniques have been used to automatically discover typical user profiles from Web access logs. But it is a challenging problem that many clustering algorithms yield poor results because the session vectors are usually high dimensional and sparse. Although standard non-negative matrix factorization (SNMF) can be used in reducing the dimensionality of the session-URL matrix, the clustering results is not precise, because the basis vectors SNMF gets are not orthogonal and usually redundancy. In this paper, we apply local nonnegative matrix factorization (LNMF), which get basis vectors as orthogonal as possible, to reduce the dimensionality of the session-URL matrix. The experiment results show that LNMF performs better than SNMF for mining typical user session profile.
\end{abstract}

\section{Introduction}

Many web servers keep a access log of their users. Analysis of these logs can provide information on how to restructure a Web site for better performance[1]. Recently, new techniques[2] have been proposed to automatically discover "typical user session profiles", which are temporally compact sequences of Web accesses users often visit.

In the context of discovering Web user profiles based on clustering, a vector space model is used to represent the user sessions by assigning each vector attribute to a given URL on the Web site. However, in the case of Web sessions, the user sessions form extremely high dimensional and sparse data matrices. The key problem lies in the fact that many clustering algorithms yield poor results because of the high dimensionality[3]. To solve this problem, one method is applying standard nonnegative matrix factorization (SNMF) method, often called NMF[5], to reduce the dimensionality of the session-URL matrix as presented in [4]

* This work was supported in part by the NSFC (60373066, 60303024), National Grand Fundamental Research 973 Program of China (2002CB312000), National Research Foundation for the Doctoral Program of Higher Education of China.]

** Corresponding author: Baowen $\mathrm{Xu}$, Department of Computer Science and Engineering, Southeast University, Nanjing, 210096, China. Email: bwxu@ seu.edu.cn 
Though the approach in[4] is effective, there are still some problems remained, among which a major one is that the SNMF do not require the base vectors be orthogonal. This may cause information redundancy and weaken the precision of results. In this paper, we apply local nonnegative matrix factorization (LNMF)[6], which get basis vectors as orthogonal as possible, to reduce the dimension of the session-URL matrix. Also, we propose a metric to measure the clustering results. The experimental results show that the LNMF performs better than SNMF in mining typical user session profiles.

\section{Mining Typical User Session Profiles}

Now, we describe all the steps of mining typical user session profiles: first cluster the URL-session vectors. Then measure the clustering results and find the best one. Third summarize typical user session profile from the best clustering result.

\subsection{Clustering the URL-Session Vectors}

First, like the steps in [4], we can get user sessions from server access logs, and form a URL-session matrix. Here, a user session is defined as accesses from the same IP address, such that the duration of elapsed time between two consecutive accesses in the session is within a prespecified threshold. Each URL in the site is assigned a unique number $i \in\{1,2, \ldots, m\}$, where $m$ is the total number of valid URLs. Thus, the $j^{\text {th }}$ user session is encoded as a $m$ dimensional vector $x_{j}=\left(x_{l j}, x_{2 j}, \ldots, x_{m j}\right)^{T}$, where $x_{i j}=1$ if $i^{\text {th }}$ URL is accessed during the $j^{t h}$ session, otherwise $x_{i j}=0$. All the $n$ user session vectors form a non-negative session-URL matrix $X=\left(x_{i j}\right)_{m \times n}$.

Second, we apply LNMF to reduce the dimensionality of the session-URL matrix $X=\left(x_{i j}\right)_{m \times n}$.LNMF finds non-negative matrices $U=\left(u_{i j}\right)_{m \ltimes r}$ and $V=\left(v_{i j}\right)_{r \times n}$ such that $X \approx U V$. Each column in $X$ is called primary vector, and its corresponding column in $V$ is called projecting vector.

Third, we use the spherical $k$-means algorithm[4] to cluster the vectors in $V$. There are four steps: i) normalize all vectors $v_{j}$ to a unit vector which is still denoted as $v_{j}$. ii) apply spherical $k$-means algorithm to cluster these vectors into $k$ partitions $\left\{\pi_{j}\right\}_{j=1}^{k}$, where each $\pi_{j}(1 \leq j \leq k)$ denotes a cluster. iii) count the number of vectors each cluster $\pi_{j}(1 \leq j \leq k)$ contains, and discard the clusters whose number of vectors are less than $q$. Then we get the clustering result $\left\{\pi_{j}\right\}_{j=1}^{\tilde{k}}$, where $\tilde{k}(\tilde{k} \leq k)$ denotes the number of left clusters. vi) repeat ii) and iii) several times, and select the best clustering result according to their relative distance, which is defined in session 2.2

\subsection{Measuring the Clustering Results}

Here we present a metric to measure the "compactness" and "distinctness" of the clusters. It is the ratio of the "average intercluster distance" to the "average intracluster distance". The formal definition of these concepts are given below: 
Definition 1. Suppose the primary vector of the $i^{\text {th }}$ and $j^{\text {th }}$ sessions are $x_{i}$ and $x_{j}$ respectively, then the distance between the two sessions are defined as:

$$
S(i, j)=1-\frac{x_{i}{ }^{T} x_{j}}{\left\|x_{i}\right\| \cdot\left\|x_{j}\right\|}
$$

Definition 2. Given a clustering result $P=\left\{\pi_{j}\right\}_{j=1}^{\tilde{k}}$, its intracluster pair set $D_{1}(P)$ and its intercluster pair set $D_{2}(P)$ are defined as:

$$
\begin{gathered}
D_{1}(P)=\left\{\left(i_{1}, i_{2}\right) \mid v_{i_{1}}, v_{i_{2}} \in \pi_{n}, i_{1}<i_{2}, n=1 . . \tilde{k}\right\} \\
D_{2}(P)=\left\{\left(i_{1}, i_{2}\right) \mid v_{i_{1}} \in \pi_{n}, v_{i_{2}} \in \pi_{m}, i_{1}<i_{2}, n, m=1 . . \tilde{k}, n \neq m\right\}
\end{gathered}
$$

Suppose $i$ and $j(i<j)$ are two session numbers. " $(i, j) \in D_{1}$ " means the $i^{\text {th }}$ and the $j^{\text {th }}$ session are in the same cluster. " $(i, j) \in D_{2}$ ” means they are in different clusters.

Definition 3. Given a clustering result $P=\left\{\pi_{j}\right\}_{j=1}^{\tilde{k}}$, let $D_{1}(P)$ and $D_{2}(P)$ denote its intracluster pair set and intercluster pair set respectively, and $n_{1}, n_{2}$ denotes the number of pairs in $D_{1}(P)$ and $D_{2}(P)$, then the average intracluster distance $L_{l}(P)$ and the average intercluster distance $L_{2}(P)$ are defined as follows, where $S\left(i_{1}, i_{2}\right)$ denote the distance between the $i^{\text {th }}$ and $j^{\text {th }}$ session:

$$
\begin{array}{r}
L_{1}(P)=\frac{\sum_{\left(i_{1}, i_{2}\right) \in D_{1}(P)} S\left(i_{1}, i_{2}\right)}{n_{1}} \\
L_{2}(P)=\frac{\sum_{\left(i_{1}, i_{2}\right) \in D_{2}(P)} S\left(i_{1}, i_{2}\right)}{n_{2}}
\end{array}
$$

Definition 4. The metric relative distance $R D(P)$ is as follows:

$$
R D(P)=L_{1}(P) / L_{2}(P)
$$

Clusters in a clustering results with low relative distance are tight, compact and well-separated from others, and can be seen better than the ones with high relative distance.

\subsection{Summarizing Typical User Session Profile}

Suppose we got the best clustering result, which contain $k$ clusters $\left\{\pi_{j}\right\}_{j=1}^{k}$, here we present a method to summarize typical user session profile from each cluster.

Let $\pi_{i}=\left\{v_{1}^{i}, v_{2}^{i}, \cdots, v_{l}^{i}\right\}$, be the $i^{\text {th }}$ cluster, where $v_{j}^{i}=\left(v_{1 j}{ }^{i}, v_{2 j}{ }^{i}, \ldots, v_{r j}^{i}\right)^{T}$, whose corresponding primary vector is $x_{j}^{i}=\left(x_{1 j}{ }^{i}, x_{2 j}{ }^{i}, \ldots, x_{m j}{ }^{i}\right)^{T}$. We use the primary vectors to construct a $m$ dimensional vector $P_{i}=\left(P_{1 i}, P_{2 j}, \ldots, P_{m i}\right)^{T}$ as follows: 


$$
P_{i}=\frac{1}{l} \sum_{j=1}^{l} \frac{x_{j}^{i}}{\left\|x_{j}^{i}\right\|}
$$

Generally the components of $P_{i}$ represent the probability of access of each URL during the profile, where $P_{k i}$ measure the significance of the $k^{\text {th }}$ URL to the profile.

Table 1. Clustering results( SNMF)

\begin{tabular}{|c|c|c|c|c|c|}
\hline $\boldsymbol{r}$ & 10 & 20 & 30 & 40 & 50 \\
\hline $\boldsymbol{n}$ & 17 & 18 & 14 & 13 & 11 \\
\hline $\boldsymbol{R D}$ & 0.483 & 0.394 & 0.289 & 0.381 & 0.277 \\
\hline $\boldsymbol{r}$ & 60 & 70 & 80 & 90 & 100 \\
\hline $\boldsymbol{n}$ & 12 & 10 & 9 & 9 & 9 \\
\hline $\boldsymbol{R D}$ & 0.297 & 0.292 & 0.238 & 0.193 & 0.247 \\
\hline $\boldsymbol{r}$ & 110 & 120 & 130 & 140 & 150 \\
\hline $\boldsymbol{n}$ & 11 & 7 & 10 & 5 & 10 \\
\hline $\boldsymbol{R D}$ & 0.135 & 0.109 & 0.107 & 0.149 & 0.127 \\
\hline
\end{tabular}

Table 2. Clustering results( SNMF)

\begin{tabular}{|c|c|c|c|c|c|}
\hline $\boldsymbol{r}$ & 10 & 20 & 30 & 40 & 50 \\
\hline $\boldsymbol{n}$ & 20 & 17 & 15 & 11 & 11 \\
\hline $\boldsymbol{R D}$ & 0.500 & 0.393 & 0.170 & 0.124 & 0.095 \\
\hline $\boldsymbol{r}$ & 60 & 70 & 80 & 90 & 100 \\
\hline $\boldsymbol{n}$ & 9 & 6 & 8 & 8 & 6 \\
\hline $\boldsymbol{R D}$ & 0.066 & 0.046 & 0.062 & 0.073 & 0.047 \\
\hline $\boldsymbol{r}$ & 110 & 120 & 130 & 140 & 150 \\
\hline $\boldsymbol{n}$ & 7 & 7 & 9 & 8 & 8 \\
\hline $\boldsymbol{R D}$ & 0.046 & 0.049 & 0.057 & 0.065 & 0.055 \\
\hline
\end{tabular}

Table 3. Typical User Session Profile

\begin{tabular}{|c|c|}
\hline 1 & $\begin{array}{l}\text { 0.686-/music/machines/Analogue-Heaven } \\
0.686-/ m a c h i n e s / A n a l o g u e-H e a v e n \\
0.026-/ m u s i c / m a c h i n e s / A n a l o g u e-H e a v e n / e m a i l . h t m l\end{array}$ \\
\hline 2 & 0.964-/music/machines/Analogue-Heaven \\
\hline 3 & $\begin{array}{l}0.670-/ \text { music/machines } \\
0.670-/ \text { machines } \\
0.049-/ \text { music/machines/guide } \\
0.019-/ \text { machines/manufacturers } \\
0.018-/ \text { music/machines/manufacturers/Moog/Modular }\end{array}$ \\
\hline 4 & 0.964-/music/machines \\
\hline 5 & 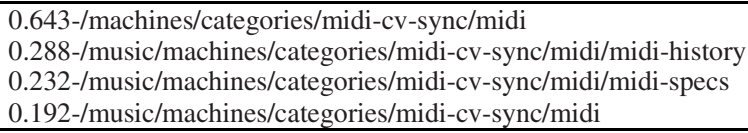 \\
\hline 6 & $\begin{array}{l}\text { 0.964-/music/machines } \\
0.901-/ \text { music/machines/samples.html } \\
0.067-/ \text { music/machines/manufacturers/ARP/Odyssey/samples } \\
0.026-/ \text { music/machines/manufacturers/Roland/SH-synths/samples } \\
0.025-/ \text { music/machines/manufacturers/Roland/JX/samples } \\
0.025-/ \text { music/machines/manufacturers/Roland/TR-606/samples } \\
0.025 \text {-/music/machines/links }\end{array}$ \\
\hline 7 & $\begin{array}{l}0.688-/ \text { machines } \\
0.688-/ \text { music/machines } \\
0.025-/ \text { music/machines/samples.html } \\
0.021-/ \text { music/machines/features }\end{array}$ \\
\hline
\end{tabular}




\section{Experimental Results}

In our experiments, we use the log data during a period of $1 / 1 / 98$ to $6 / 1 / 98$ downloaded from http://www.cs.washington.edu/research/adaptive. First, we set the maximum elapse time of a session to 45 minutes and totally get 449 valid URLs and 7577 sessions from the logs, for these URLs and sessions, we get the session-URL matrix $X$. Then we apply the SNMF and LNMF respectively to reduce the high dimension of the session-URL matrix $X$. Third, for each projecting matrix $V$, we set $k=400$ and $q=100$ and get the all clustering results, listed in table 1 and 2 . At last we compare these clustering results, and mine typical user session profiles from the best one, which are listed in table 3.

Table 1 and Table 2 show the cluster numbers $n$ and the relative distance $R D$ of the clustering results based on SNMF and LNMF with different projecting vector dimension $r$. From the tables, we can see that value of $n$ fluctuate wildly in table 1 , while in table 2 numbers is more stable. Also the relative distance is much smaller in table 2 than in table 1 , this means that the clustering results in table 2 is more precise. The reason of the difference may be that the SNMF makes the basis matrix $U$ generated more randomly and cause information redundancy. So that the clustering results based on SNMF fluctuate wildly and have less precision. While the LNMF adds more additional constrains on the basis vectors and solves these problems. From this, we get the conclusion that the cluster method based on LNMF is more suitable in mining typical session profile.

\section{Conclusions}

In this paper, LNMF is proposed instead of SNMF in the field of mining typical user session profiles. The experimental results show that the method based on LNMF performs better than the method based on SNMF. In the future we will take more consideration into the impact of other constraints in this field.

\section{References}

1. Cooley, R., Mobasher, B., Srivastava, J.: Web mining: Information and Pattern discovery on the World Wide Web. Proceeding. of International Conference on Tools with Artificial Intelligence, Newport beach, USA (1997) 558-567

2. Nasraoui, O., Frigui, H., Krishnapuram, R.: Extracting Web User Profiles Using Relational Competitive Fuzzy Clustering. Internatiol Journal on Artifical Intelligence Tools, 9(4) (2000) 509-526

3. Hanm, J., Kamber., M.: Data Mining: Concepts and Techniques. Morgan Kaufmann Publishers (2000)

4. Lu, J.J., Xu, B.W., Yang, H.J.: Matrix dimensionality reduction for mining Web access logs. Proceeding of IEEE/WIC International Conference on Web Intelligence, Halifax, CA (2003) 405-408

5. Lee, D.D., Seung, H.S.: Learning the parts of objects by non-negative matrix factorization. Nature 401 (1999) 788-791

6. Li, S.Z., Hou, X.W., Zhang, H.J.: Learning spatially localized parts-based representation. Proceeding of the CVPR'01 Conference, Hawaii, USA (2001) 207-212 